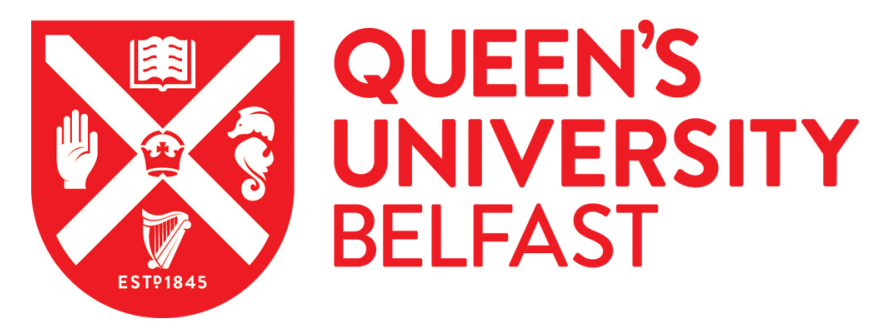

\title{
Dynamic Multigrain Parallelization on the Cell Broadband Engine
}

Blagojevic, F., Nikolopoulos, D., Stamatakis, A., \& Antonopoulos, C. D. (2007). Dynamic Multigrain Parallelization on the Cell Broadband Engine. In Proceedings of the 12th ACM SIGPLAN Symposium on Principles and Practice of Parallel Programming (PPOPP): Best Paper Award (pp. 90-100). ACM. https://doi.org/10.1145/1229428.1229445

Published in:

Proceedings of the 12th ACM SIGPLAN Symposium on Principles and Practice of Parallel Programming (PPOPP)

Queen's University Belfast - Research Portal:

Link to publication record in Queen's University Belfast Research Portal

\section{General rights}

Copyright for the publications made accessible via the Queen's University Belfast Research Portal is retained by the author(s) and / or other copyright owners and it is a condition of accessing these publications that users recognise and abide by the legal requirements associated with these rights.

Take down policy

The Research Portal is Queen's institutional repository that provides access to Queen's research output. Every effort has been made to ensure that content in the Research Portal does not infringe any person's rights, or applicable UK laws. If you discover content in the Research Portal that you believe breaches copyright or violates any law, please contact openaccess@qub.ac.uk. 


\section{Dynamic Multigrain Parallelization on the Cell Broadband Engine}

\author{
Filip Blagojevic, \\ Dimitrios S. Nikolopoulos \\ Center for High-End Computing Systems \\ and Department of Computer Science \\ Virginia Tech \\ 660 McBryde Hall \\ Blacksburg, VA 24061 \\ $\{$ filip,dsn $\} @ c s . v t . e d u$
}

\author{
Alexandros Stamatakis \\ School of Computer \& \\ Communication Sciences \\ École Polytechnique \\ Fédérale de Lausanne \\ Station 14, Ch-1015 Lausanne, \\ Switzerland \\ Alexandros.Stamatakis@epfl.ch
}

\author{
Christos D. Antonopoulos \\ Department of Computer Science \\ College of William and Mary \\ McGlothlin-Street Hall \\ Williamsburg, VA 23187-8795 \\ and Division of Research and Informatics \\ Greek Armed Forces \\ cda@cs.wm.edu
}

\begin{abstract}
This paper addresses the problem of orchestrating and scheduling parallelism at multiple levels of granularity on heterogeneous multicore processors. We present mechanisms and policies for adaptive exploitation and scheduling of layered parallelism on the Cell Broadband Engine. Our policies combine event-driven task scheduling with malleable loop-level parallelism, which is exploited from the runtime system whenever task-level parallelism leaves idle cores. We present a scheduler for applications with layered parallelism on Cell and investigate its performance with RAxML, an application which infers large phylogenetic trees, using the Maximum Likelihood (ML) method. Our experiments show that the Cell benefits significantly from dynamic methods that selectively exploit the layers of parallelism in the system, in response to workload fluctuation. Our scheduler outperforms the MPI version of RAxML, scheduled by the Linux kernel, by up to a factor of 2.6. We are able to execute RAxML on one Cell four times faster than on a dual-processor system with Hyperthreaded Xeon processors, and 5-10\% faster than on a single-processor system with a dual-core, quad-thread IBM Power5 processor.
\end{abstract}

Categories and Subject Descriptors D.1.3 [Programming Techniques]: Concurrent Programming- Parallel programming; D.4.1 [Operating Systems]: Process Management- Scheduling; C.4 [Performance of Systems]: -Design studies.

General Terms Design, Management, Performance.

Keywords IBM Cell, Programming Models, Process Scheduling, RAxML.

\section{Introduction}

In the quest for delivering higher performance to scientific applications, hardware designers have began to move away from conventional superscalar processors and have embraced architectures with multiple cores. Although most vendors are already marketing

Permission to make digital or hard copies of all or part of this work for personal or classroom use is granted without fee provided that copies are not made or distributed for profit or commercial advantage and that copies bear this notice and the full citation on the first page. To copy otherwise, to republish, to post on servers or to redistribute to lists, requires prior specific permission and/or a fee.

PPoPP '07 March 14-17, 2007, San Jose, California.

Copyright (C) 2007 ACM 978-1-59593-602-8/07/0003 .. \$5 .00 multicore processors, these processors are largely based on replication of simple scalar or superscalar cores. Unfortunately, such designs exhibit some well-known limitations, in both performance and power. These limitations, in conjunction with the sustained demand for higher performance, stimulated interest in unconventional designs, utilizing heterogeneous cores and specialized accelerators on the same chip. The Cell Broadband Engine (BE) is a representative of these designs, which has recently drawn considerable attention by industry and academia. Since it was originally designed for the game box market, Cell has low cost and a modest power budget. Nevertheless, the processor is able to achieve unprecedented peak single-precision floating point performance, a property which makes Cell suitable for high-end computing. IBM announced recently the development of a new Petaflop system with 16,000 Cell CPUs, due for delivery in 2008.

The potential of the Cell BE has been demonstrated in a number of recent studies $[8,21,27]$. The Cell has eight high-frequency specialized execution cores with pipelined SIMD capabilities, and an aggressive data transfer architecture. The processor achieves a theoretical peak performance of over 200 Gflops for single-precision FP calculations and has a peak memory bandwidth of over $25 \mathrm{Gi}$ gabytes/s. These performance figures position Cell ahead of the competition against even the most powerful general-purpose multicore microprocessors. Cell has already demonstrated impressive performance ratings in applications and computational kernels with highly vectorizable data parallelism, such as signal processing, compression, encryption, dense and sparse numerical kernels [8, $11,22]$.

This paper explores Cell from a different perspective, namely that of multigrain parallelization. Cell is quite unique as a processor, in that it can exploit orthogonal dimensions of task and data parallelism on a single chip. The processor includes an SMT Power Processing Element (PPE) and 8 Synergistic Processing Elements (SPEs). The PPE can serve as a general purpose multithreaded execution engine, or as a scheduler of computations off-loaded to SPEs. The SPEs themselves provide the bulk of the Cell's computational power, thanks to their pipelined SIMD architecture.

A programmer is faced with a seemingly vast number of options while developing parallel code on Cell. Functional and data decompositions of the program can be implemented on both the PPE and the SPEs. Typically, the programmer is expected to place the workload almost entirely on SPEs for higher performance. Functional decompositions can be achieved by splitting tasks between the PPE and the SPEs and off-loading tasks from the PPE to SPEs at runtime. Data decompositions may exploit the vector units of 
the SPEs, or exploit parallelism at two levels, using loop decomposition across SPEs and vectorization within SPEs. Functional and data decompositions can be static or dynamic, and they should be orchestrated carefully to fully utilize the SPEs. Although the Cell vendors already provide programming support for using some of the aforementioned parallelization strategies, selecting the most effective strategy and actually combining and scheduling layered parallelism on Cell can be an arduous task for the programmer.

To simplify programming and improve efficiency on Cell, we present a set of dynamic scheduling policies and the associated mechanisms. The purpose of these policies is to allow the application to exploit the proper layers and degrees of parallelism, in order to maximize efficiency on the Cell's computational cores, including both the SPEs and the PPE. We explore the design and implementation of our scheduler using RAxML [26] as a case study. RAxML is a computational biology application which infers large phylogenetic trees, using the Maximum Likelihood (ML) criterion. The RAxML algorithm is extremely computationally intensive. The code is embarrassingly parallel at the task-level and exhibits intrinsic loop-level parallelism within each task. Therefore, it is a good candidate for parallelization on Cell. For a real world biological analysis, RAxML typically needs to execute between 20 and 200 distinct tree searches and between 100 and 1,000 bootstrapped analyses. Each of those searches represents an independent task, with single-nested loops that are both parallelizable and vectorizable. This paper makes the following contributions:

- We present a runtime system and scheduling policies that exploit polymorphic (task and loop-level) and layered parallelism on Cell. Our runtime system is adaptive, in the sense that it chooses the form and degree of parallelism to expose to the hardware, in response to workload fluctuation. Since the right choice of form(s) and degree(s) of parallelism depends nontrivially on the workload and on the input, our runtime system unloads an important burden from the programmer.

- We show that dynamic multigrain parallelization is a necessary optimization for sustaining maximum performance on Cell, since no static parallelization scheme is able to achieve high SPE efficiency in all cases.

- We present an event-driven multithreading execution engine, which achieves higher efficiency on SPEs by oversubscribing the PPE.

- We present a feedback-guided scheduling policy for dynamically triggering and throttling loop-level parallelism across SPEs on Cell. We show that work-sharing of divisible tasks across SPEs should be used when the event-driven multithreading engine of the PPE leaves half or more of the SPEs idle. We observe benefits from loop-level parallelization of off-loaded tasks across SPEs. However, we also observe that loop-level parallelism should be exposed only in conjunction with lowdegree task-level parallelism. Its effect diminishes as the degree of task-level parallelism in the application increases at runtime.

To put our study in a broader context, we present comparisons of the Cell BE against a dual-processor SMP system with Hyperthreaded Xeon processors and against a single-processor IBM Power5-based system. The Power5 is a leading dual-core processor with 2-way SMT cores. We observe that Cell outperforms both platforms. Taking into account cost and power efficiency, Cell exhibits great promise as the processor of choice for high-end systems and challenging applications.

The rest of this paper is organized as follows: Section 2 provides a brief overview of recent work on Cell. Section 3 describes RAxML. Section 4 outlines the architecture of Cell. Section 5 presents our runtime system and scheduling policies, along with their experimental evaluation. Section 6 summarizes the paper.

\section{Related Work}

We briefly summarize published research on Cell, which includes performance analysis of various aspects of the processor, various compiler/runtime support environments and a few application case studies.

Kistler et. al [17] present results on the performance of the Cell's on-chip interconnection network. They show a series of experiments that estimate the DMA latencies and bandwidth of Cell, using microbenchmarks. They also investigate the system behavior under different patterns of communication between local storage and main memory. Williams et. al [28] present an analytical framework to predict performance on Cell. In order to test their model, they use several computational kernels, including dense matrix multiplication, sparse matrix vector multiplication, stencil computations, and 1D/2D FFTs. In addition, they propose microarchitectural modifications that can increase the performance of Cell when operating on double-precision floating point elements. Our work considers the performance implications of multigrain parallelization strategies on Cell, using a real-world parallel application from the area of computational biology.

Eichenberger et. al [11] present several compiler techniques targeting automatic generation of highly optimized code for Cell. These techniques attempt to exploit two levels of parallelism, thread-level and SIMD-level, on the SPEs. The techniques include compiler assisted memory alignment, branch prediction, SIMD parallelization, OpenMP thread-level parallelization, and compilercontrolled software caching. The study of Eichenberger et. al. does not present details on how multiple levels of parallelism are exploited and scheduled simultaneously by the compiler, or similar guidelines for the programmer. Scheduling layered and polymorphic parallelism is the central theme of this paper. The compiler techniques presented in [11] are also complementary to the work presented in this paper. They focus primarily on extracting high performance out of each individual SPE, whereas our work focuses on scheduling and orchestrating computation across SPEs.

Although Cell has been a focal point in numerous articles in popular press, published research using Cell for real-world HPC applications beyond games is scarce. Hjelte [16] presents an implementation of a smooth particle hydrodynamics simulation on Cell. This simulation requires good interactive performance, since it lies on the critical path of real-time applications such as interactive simulation of human organ tissue, body fluids, and vehicular traffic. Benthin et. al [4] present an implementation of ray-tracing algorithms on Cell, aiming also at high interactive performance. Bader et. al [3] present an algorithmic model of Cell and use the model to implement an efficient index ranking algorithm.

\section{RAxML-VI-HPC}

RAxML-VI-HPC (v2.1.3) (Randomized Axelerated Maximum Likelihood version VI for High Performance Computing) [26] is a program for large-scale ML-based (Maximum Likelihood [13]) inference of phylogenetic (evolutionary) trees using multiple alignments of DNA or AA (Amino Acid) sequences. The program is freely available as open source code at http://icwww.epfl.ch/ stamatak (software frame).

Phylogenetic trees are used to represent the evolutionary history of a set of $n$ organisms. An alignment with the DNA or AA sequences representing those $n$ organisms (also called taxa) can be used as input for the computation of phylogenetic trees. In a phylogeny the organisms of the input data set are located at the tips (leaves) of the tree whereas the inner nodes represent extinct 
common ancestors. The branches of the tree represent the time which was required for the mutation of one species into another, new one. The inference of phylogenies with computational methods has many important applications in medical and biological research (see [2] for a summary). An example for the evolutionary tree of the monkeys and the homo sapiens is provided in Figure 1.

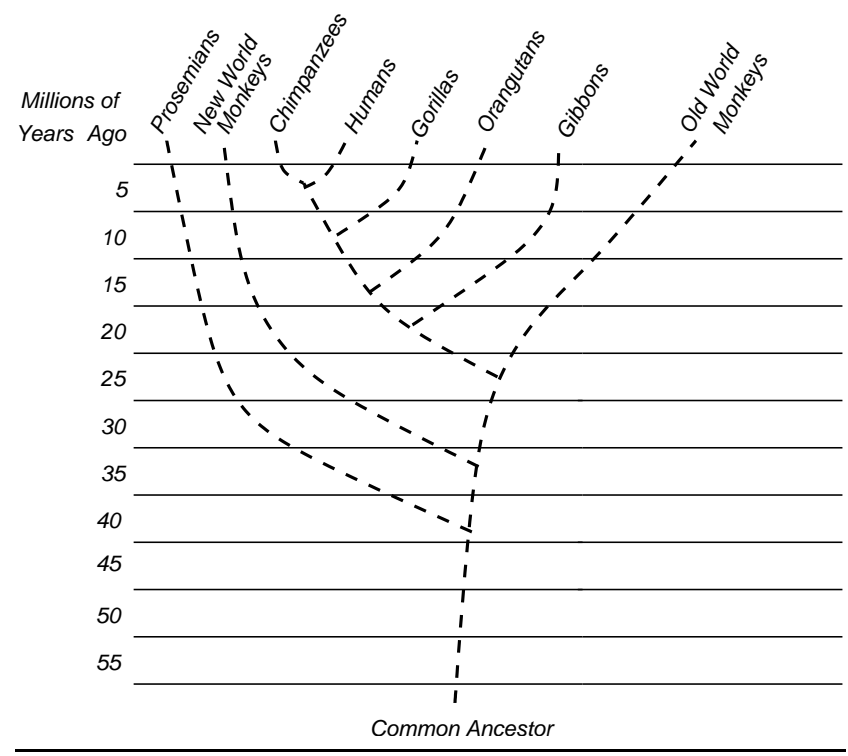

Figure 1. Phylogenetic tree representing the evolutionary relationship between monkeys and the homo sapiens.

Due to the rapid growth of sequence data over the last years, it has become feasible to compute large trees which often comprise more than 1,000 organisms and sequence data from several genes (so-called multi-gene alignments). This means that alignments grow in the number of organisms as well as in sequence length. The computation of the tree-of-life containing representatives of all living beings on earth is still one of the grand challenges in Bioinformatics.

The fundamental algorithmic problem in computational phylogeny is the immense amount of alternative tree topologies which grows exponentially with the number of organisms $n$, e.g. for $n=50$ organisms there exist $2.84 * 10^{76}$ alternative trees. As a comparison, the number of atoms in the universe is approximately $10^{80}$. In fact, it has only recently been shown that the ML phylogeny problem is NP-hard [9]. In addition, ML-based inference of phylogenies is very memory- and floating point-intensive, such that the application of high performance computing techniques and new parallel processor architectures can contribute significantly to the reconstruction of larger and more accurate trees.

Nonetheless, over the last years there has been significant progress in the field of heuristic ML search algorithms with the release of programs such as IQPNNI [20], PHYML [15], GARLI [29] and RAxML [24, 26].

Some of the largest published ML-based biological analyses to date have been conducted with RAxML [14, 18, 19, 23]. The program is also part of the greengenes project [10] (greengenes.lbl.gov) as well as the CIPRES (CyberInfrastructure for Phylogenetic RESearch, www.phylo.org) project. To the best of the authors' knowledge RAxML-VI-HPC has been used to compute trees on the two largest data matrices analyzed under ML to date: a 25,057-taxon alignment of protobacteria (length: 1,463 nucleotides) and a 2,182-taxon alignment of mammals (length: 51,089 nucleotides).
The current version of RAxML incorporates a significantly improved rapid hill climbing search algorithm. A recent performance study [26] on real world datasets with $\geq 1,000$ sequences reveals that it is able to find better trees in less time and with lower memory consumption than other current ML programs (IQPNNI, PHYML, GARLI). Moreover, RAxML-VI-HPC has been parallelized with MPI (Message Passing Interface), to enable embarrassingly parallel non-parametric bootstrapping and multiple inferences on distinct starting trees in order to search for the best-known ML tree (see Section 3.1 for details). In addition, it has been parallelized with OpenMP [25]. Like every ML-based program, RAxML exhibits a source of fine-grained loop-level parallelism in the likelihood functions which consume over $90 \%$ of the overall computation time. This source of parallelism scales particularly well on large memory-intensive multi-gene alignments due to increased cache efficiency. Finally, RAxML has also recently been ported to a GPU (Graphics Processing Unit) [7].

\subsection{The MPI Version of RAxML}

The MPI version of RAxML is the basis for its porting and further parallelization on Cell. The MPI code exploits the embarrassing parallelism that is inherent to every real-world phylogenetic analysis. In order to conduct a "publishable" tree reconstruction a certain number (typically 20-200) of distinct inferences (tree searches) on the original alignment as well as a large number (typically 1001,000) of bootstrap analyses have to be conducted (see [14] for an example of a real-world analysis with RAxML). Thus, if the dataset is not extremely large, this represents the most reasonable approach to exploit HPC platforms from a user's perspective.

Multiple inferences on the original alignment are required in order to determine the best-known (best-scoring) ML tree (we use the term best-known because the problem is NP-hard). This is the tree which will then be visualized and published. In the case of RAxML, each independent tree search starts from a distinct starting tree. This means, that the vast topological search space is traversed from a different starting point every time and will yield final trees with different likelihood scores. For details on the RAxML search algorithm and the generation of starting trees, the reader is referred to [24].

Bootstrap analyses are required to assign confidence values ranging between 0.0 and 1.0 to the internal branches of the bestknown ML tree. This allows to determine how well-supported certain parts of the tree are and is important for the biological conclusions drawn from it. Bootstrapping is essentially very similar to multiple inferences. The only difference is that inferences are conducted on a randomly re-sampled alignment for every bootstrap run, i.e. a certain amount of columns (typically 10-20\%) is reweighted. This is performed in order to assess the topological stability of the tree under slight alterations of the input data. For a typical biological analysis, a minimum of 100 bootstrap runs is required.

All those individual tree searches be it bootstrap or multiple inferences, are completely independent from each other and can thus be exploited by a simple master-worker scheme.

\section{The Cell Broadband Engine}

The main components of the Cell BE are a multithreaded Power Processing element (PPE) and eight Synergistic Processing Elements (SPEs) [12]. These elements are connected with an on-chip Element Interconnect Bus (EIB). The SPEs are placed in equal distances around the bus.

The PPE is a 64-bit, dual-thread PowerPC processor, with Vector/SIMD Multimedia extensions [1] and two levels of on-chip cache. The L1-I and L1-D caches have a capacity of $32 \mathrm{~KB}$, while the $\mathrm{L} 2$ cache has a capacity of $512 \mathrm{~KB}$. In this work we use a Cell 
blade with two Cell BEs running at $3.2 \mathrm{GHz}$, and $1 \mathrm{~GB}$ of XDR RAM (512 MB per processor). The PPEs run Linux Fedora Core 5. We use the Toolchain 4.0.2 compilers.

The SPEs are the primary computing engines of the Cell processor. Each SPE is a 128-bit processor with two major components: a Synergistic Processor Unit (SPU) and a Memory Flow Controller (MFC). All instructions are executed on the SPU. The SPU includes 128 registers, each 128 bits wide, and $256 \mathrm{~KB}$ of softwarecontrolled local storage. The SPU can fetch instructions and data only from its local storage and can write data only to its local storage. The SPU implements a Cell-specific set of SIMD instructions. All single precision floating point operations on the SPU are fully pipelined, and the SPU can issue one single-precision floating point operation per cycle. Double precision floating point operations are partially pipelined and two double-precision floating point operations can be issued every six cycles. Double-precision FP performance is therefore significantly lower than single-precision FP performance. With all eight SPUs active and fully pipelined double precision FP operation, the Cell BE is capable of a peak performance of 21.03 Gflops. In single-precision FP operation, the Cell $\mathrm{BE}$ is capable of a peak performance of 230.4 Gflops [8].

The SPE can access RAM through direct memory access (DMA) requests. The DMA transfers are handled by the MFC. All programs running on an SPE use the MFC to move data and instructions between local storage and main memory. Data transferred between local storage and main memory must be 128-bit aligned. The size of each DMA transfer can be at most $16 \mathrm{~KB}$. DMA-lists can be used for transferring large amount of data (more than $16 \mathrm{~KB}$ ). A list can have up to 2,048 DMA requests, each for up to $16 \mathrm{~KB}$. The MFC supports only DMA transfer of blcks that are $1,2,4,8$ or multiples of 16 bytes long.

The EIB is an on-chip coherent bus that handles communication between the PPE, SPE, main memory, and I/O devices. The EIB is a 4-ring structure, and can transmit 96 bytes per cycle, for a bandwidth of 204.8 Gigabytes/second. The EIB can support more then 100 outstanding DMA requests.

\section{Scheduling and Runtime Support for Multigrain Parallelization on Cell}

In this section we present our scheduling policies and their implementation as a runtime environment on Cell. We use RAxML to evaluate the policies. We first discuss briefly the optimization of RAxML bootstraps for the Cell SPEs (Section 5.1). We discuss our event-driven multithreading scheduler for task-level parallelization in Section 5.2. In Section 5.3, we discuss our adaptive loop scheduler and its implementation. Section 5.4 presents policies and mechanisms to adaptively merge task-level and loop-level parallelism.

\subsection{SPE Optimizations}

A straightforward adaptation of the MPI version of RAxML on Cell is to execute multiple MPI processes on the PPE and to have the major computational kernels of each process offloaded to an SPE. Each MPI process executes one RAxML bootstrap at a time. To identify the parts of RAxML that are suitable for SPE execution, we profiled the code executed by an MPI process using the gprof profiler. For all profiling and production run experiments presented in this paper, we used the file 42_SC as input to RAxML. 42_SC contains 42 organisms. Each organism is represented by a DNA sequence of 1167 nucleotides.

On an IBM Power processor, $98.77 \%$ of the execution time of RAxML is spent in three functions which compute the likelihood: $76.8 \%$ of execution time is spent in newview (), $2.37 \%$ of execution time is spent in evaluate (), and $19.6 \%$ of execution time is spent in makenewz (). These functions are the obvious candidates for off-loading to SPEs. We off-load the functions as a group, in a single code module loaded on each SPE. The advantage of having a single module is that it can be loaded to the local storage of each SPE once and reused throughout the execution of the application, unless a change in the degree or form of parallelism executed on the SPEs is dictated by the runtime system. This observation is valid under the assumption that the code of all three functions fits in the local storage of each SPE and still leaves enough space for allocating the stack, data and buffers.

The drawback of merging off-loaded functions is that the larger size of the code module reduces the space available on the SPE for the stack and heap segments. In the case of RAxML, when all three functions are off-loaded, the total size of the code segment in the off-loaded file is $117 \mathrm{~KB}$. The remaining space in the local storage $(139 \mathrm{~KB})$ is sufficient for the stack and heap segments, since the working set of the SPE functions is small for realistic problem sizes. In the general case, off-loading should be controlled dynamically to achieve a good trade-off between code locality, data locality and overall performance.

Naïve off-loading has a negative effect on performance for RAxML. We measure the execution time of RAxML before and after the three dominant functions are off-loaded, using one thread on the PPE and one SPE. The execution time of RAxML before off-loading any function to an SPE with the 42_SC input is 38.23s. The execution time after off-loading the three functions increases to $50.38 \mathrm{~s}$. There are several reasons for the observed performance degradation:

- The off-loaded code is working on double-precision floating point numbers, and the double-precision FP operations are neither vectorized, nor fully pipelined in the original code.

- The SPE achitecture does not include dynamic branch prediction. In the off-loaded code, $45 \%$ of the execution time is spent in condition checking, and the inherently random distribution of branch targets in the code makes the outcome of the conditions hard to predict.

- The DMA transfers between the local storage and the main memory are not optimized.

- The code uses expensive mathematical functions such as $\log ()$ and $\exp ()$.

- The communication between the PPE and the SPEs is not optimized.

We used this itemized list as a guideline for optimizing the off-loaded code of RAxML on Cell. We implemented vectorization of the ML calculation loops and vectorization of conditionals. We pipelined the vector operations, aggregated data transfers and replaced the original mathematical functions with numerical approximations of the same functions from the Cell SDK library. The specifics of these optimizations are beyond the scope of this paper. A detailed description is provided elsewhere [5]. The execution time of the optimized SPE code of RAxML was reduced from $50.38 \mathrm{~s}$ to $28.82 \mathrm{~s}$, which corresponds to a speedup of 1.32 over single-threaded execution on the PPE.

\subsection{Scheduling Task-Level Parallelism}

Mapping MPI code on Cell can be achieved by assigning one MPI process to each thread of the PPE. Given that the PPE is a dual-thread engine, MPI processes on the PPE can utilize two out of the eight SPEs via concurrent function off-loading. We consider two strategies to use the rest of the SPEs on Cell. The first is multi-level parallelization, and more specifically loop-level parallelization within the off-loaded functions and loop distribution 
across SPEs. The second is a model for event-driven task-level parallelism, in which the PPE scheduler oversubscribes the PPE with more than two MPI processes, to increase the availability of computation for the SPEs.

We first examine the event-driven task parallelization model, since it provides an opportunity for coarse-grained parallelization. We will refer to this model as EDTLP, for event-driven task-level parallelism, in the rest of the paper. In EDTLP, tasks correspond to off-loadable functions from MPI processes running concurrently or in a time-shared manner on the PPE. These tasks are served by running MPI processes using a fair sharing algorithm, such as round-robin. The scheduler off-loads a task immediately upon request from an MPI process, and switches to another MPI process while off-loading takes place. Switching upon off-loading prevents MPI processes from blocking the code while waiting for their tasks to complete on SPEs.

EDTLP can be implemented using a user-level scheduler to interleave off-loading across MPI processes. The scheduler is simple to implement and it can be integrated transparently in the original MPI code, provided that the tasks that need to be off-loaded are annotated. EDTLP overcomes the problem of crippling underutilization of the cores, both PPEs and SPEs, when PPE threads do not have tasks to off-load, or when PPE threads wait for completion of already off-loaded tasks.

Multiplexing more than two MPI processes on the PPE introduces system overhead due to context switching and due to implicit costs following context-switching across address spaces, such as cache and TLB pollution. Furthermore, the granularity of the off-loaded code is critical as to whether the multiplexing cost can be tolerated or not. The off-loaded code should be coarse enough to mask the overhead of multiplexing. Our EDTLP scheduler uses granularity control and voluntary context switches to address these issues.

Formally, the EDTLP scheduler executes a task graph comprising PPE tasks and SPE tasks. The scheduler follows a dependencedriven execution model. If the scheduler locates a task to be offloaded in an MPI process, it searches for an idle SPE, and if it finds one, it sends a signal to begin task execution. If no idle SPE is found, the scheduler waits until an SPE becomes available. Let $t_{\text {spe }}$ denote the execution time of a task on an SPE, $t_{\text {code }}$ denote the time needed to load the code of a task on the SPE, and $t_{\text {comm }}$ denote the time to send a signal from the PPE to an SPE to commence execution of an off-loaded task, or vice versa, to send a result from an SPE back to the PPE. The scheduler selects to off-load tasks that meet the condition $t_{\text {spe }}+t_{\text {code }}+2 \cdot t_{\text {comm }}<t_{\text {ppe }}$, where $t_{\text {ppe }}$ is the execution time of the task on the PPE. Note that $t_{\text {code }}=0$ if a task is executed on an SPE more than once and for all executions of the task other than the first. Our runtime system preloads annotated SPE functions to amortize the code shipping cost. The code remains on the SPEs, unless the runtime system decides to change its parallelization strategy and either trigger or throttle loop-level parallelism. This issue is discussed further in Section 5.4.

Since the scheduler does not know the length of the tasks a-priori, it optimistically off-loads any user-designated task and throttles off-loading for tasks that do not pass the granularity test. To implement this dynamic scheme, the code needs to maintain PPE and SPE versions of off-loadable functions. This is an easy modification, since PPE implementations of all off-loadable functions are available in the original MPI code. The modification comes at the expense of an increased code footprint on the PPE. If the scheduler does not find tasks to off-load, it blocks until a new off-loading request originates from a PPE thread.

Figure 2 illustrates an example of the operation of the EDTLP scheduler. The example uses PPE and SPE task sizes which are representative of RAxML functions. We show the execution of two

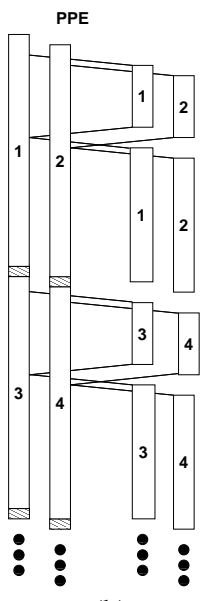

(b)

Figure 2. Scheduler behavior for two off-loaded tasks, representative of RAxML. Case (a) illustrates the behavior of our EDTLP scheduler. Case (b) illustrates the behavior of the Linux scheduler with the same workload. The numbers in the tasks correspond to MPI processes, from which the tasks originate. The shaded slots indicate context switches.

off-loaded tasks, with a 1:3 length ratio. In case (a), once a task is off-loaded by an MPI thread, the PPE switches context. At any time, two MPI threads can off-load tasks concurrently, however multiplexing the MPI threads with EDTLP enables the scheduler to use all 8 SPEs for a significant part of the coarse-grained function, and at least 4 SPEs for a significant part of the fine-grained function. In case (b), the scheduler runs persistently one MPI thread on the PPE until all functions from that task are off-loaded. The implication is that 6 out of the 8 SPEs remain idle most of the time. In RAxML, the off-loaded tasks have durations up to $96 \mu$ s. Their granularity is an order of magnitude finer than the granularity of the Linux scheduler's base time quantum, which is $10 \mathrm{~ms}$. Therefore, the OS scheduler is highly unlikely to switch context upon function off-loading. The EDTLP scheduler resolves this problem, thus achieving higher SPE utilization. The context switching overhead on the PPE is $1.5 \mu$ s per switch. This overhead is low enough to tolerate up to 7 context switches while one RAxML task is running.

We evaluate our EDTLP scheduler by comparing its performance to the performance achieved with the Linux 2.6.17 scheduler and without user-level scheduling support on our Cell blade. In this evaluation, we use the fully optimized version of RAxML, outlined in Section 5.1. This version off-loads the three ML calculation functions to SPEs. From the total execution time of one nonparametric bootstrap analysis of RAxML, $90 \%$ is spent to compute on SPEs and $10 \%$ is spent to compute and schedule tasks on the PPE. The average SPE computing time is $96 \mu$ s. The average PPE computing time between consecutive offloads is $11 \mu \mathrm{s}$.

Table 1 summarizes the results of the experiment. The first column contains the number of workers used, and the amount of work performed. RAxML is always executed in a massively parallel setting, with constant problem size (one bootstrap) per MPI process. The second column contains execution times of RAxML, when the MPI processes on the PPEs are scheduled with the EDTLP scheduler. The third column contains execution times when the MPI processes are scheduled by the Linux scheduler. Ideally, the total execution time should remain constant. The reason why this is not the case is the sub-optimal $(90 \%)$ coverage of parallel code executed on the SPEs, contention between MPI processes sharing the SMT pipeline of the PPE, and SPE parallelization and synchroniza- 


\begin{tabular}{|l|l|l|}
\hline & EDTLP & Linux \\
\hline 1 worker, 1 bootstrap & $28.46 \mathrm{~s}$ & $28.42 \mathrm{~s}$ \\
\hline 2 workers, 2 bootstraps & $29.36 \mathrm{~s}$ & $29.23 \mathrm{~s}$ \\
\hline 3 workers, 3 bootstraps & $32.54 \mathrm{~s}$ & $56.95 \mathrm{~s}$ \\
\hline 4 workers, 4 bootstraps & $33.12 \mathrm{~s}$ & $57.38 \mathrm{~s}$ \\
\hline 5 workers, 5 bootstraps & $37.27 \mathrm{~s}$ & $85.88 \mathrm{~s}$ \\
\hline 6 workers, 6 bootstraps & $38.66 \mathrm{~s}$ & $86.43 \mathrm{~s}$ \\
\hline 7 workers, 7 bootstraps & $41.87 \mathrm{~s}$ & $114.92 \mathrm{~s}$ \\
\hline 8 workers, 8 bootstraps & $43.32 \mathrm{~s}$ & $115.51 \mathrm{~s}$ \\
\hline
\end{tabular}

Table 1. Performance comparison for RAxML with different scheduling policies. The second column contains the execution times with EDTLP. The third column contains execution times with the Linux kernel scheduler. The input file is 42_SC.

tion overhead. The EDTLP scheduler maintains the execution time within a factor of 1.5 of the optimal and achieves about 2.6 times the performance of the Linux scheduler.

\subsection{Scheduling Loop-Level Parallelism}

The EDTLP model described in Section 5.2 is effective if the PPE has enough coarse-grained functions to off-load to SPEs. In cases where the degree of available task parallelism is less than the number of SPEs, the runtime system can activate a second layer of parallelism, by splitting an already off-loaded task across multiple SPEs. We implemented runtime support for parallelization of forloops enclosed within off-loaded SPE functions. We parallelize loops in off-loaded functions using work-sharing constructs similar to those found in OpenMP. In RAxML, all for-loops in the three off-loaded functions have no loop-carried dependencies, and obtain speedup from parallelization, assuming that there are enough idle SPEs dedicated to their execution. The number of SPEs activated for work-sharing is user- or system-controlled, as in OpenMP. We discuss dynamic system-level control of loop parallelism further in Section 5.4.

As an example of loop-level parallelization, we use a loop from function evaluate(), shown in Figure 3.

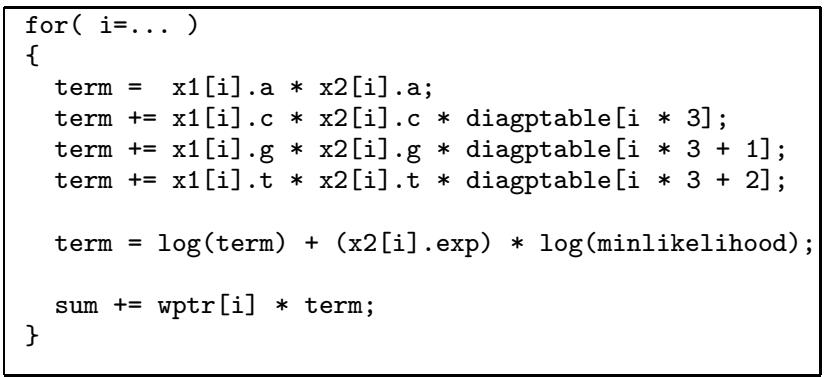

Figure 3. A parallel loop from function evaluate() of RAxML.

The basic work-sharing scheme is presented in Figure 4. Before loop execution, a designated master SPE thread sends a signal to all designated SPE worker threads. After sending the signal, the main thread executes its assigned portion of the loop. The main thread and all the workers fetch the chunk of data they need to execute their portions of the loop from the shared RAM. Global shared data modified during loop executions is committed to RAM. Data needed by the master SPE upon loop completion to make forward progress, is sent directly from the worker SPEs via SPE to SPE communication, in order to avoid the latency of communicating through shared memory. SPE to SPE communication en-

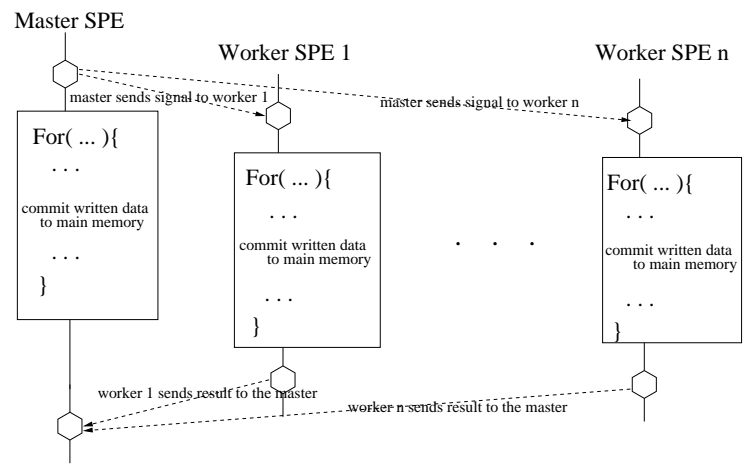

Figure 4. Parallelizing a loop across SPEs using a work-sharing model.

struct Pass\{
volatile unsigned int v1_ad;
volatile unsigned int v2_ad;
//...arguments for loop body
volatile unsigned int vn_ad;
volatile double res;
volatile int sig[2];
\}_-_attribute__((aligned $(128))) ;$

Figure 5. The data structure Pass is used for communication among SPEs. The $v_{i_{-}} a d$ variables are used to pass input arguments for the loop body from one local storage to another. The variable sig is used as a notification signal that the memory transfer for the shared data updated during the loop is completed. The variable res is used to send results back to the master SPE, and as a dependence resolution mechanism.

ables dependence-driven execution of multiple parallel loops across SPEs.

Note that the work performed by the master SPE thread, specifically loop distribution across SPEs, can also be performed by the PPE. Consequently, the PPE would also collect the final results from all SPEs involved in the loop parallelization. Collecting the results in this manner, however, increases PPE-SPE communication, especially when the distributed loop is a multi-level nested loop. Unnecessary PPE-SPE communication can be harmful for performance [5]. In order to off-load as much work to SPEs as possible [6], and to reduce PPE-SPE communication, we select an SPE to distribute loops to and collect the results from other SPEs.

In the example in Figure 3, the SPEs first perform a local reduction. The master SPE accumulates the sum received from worker SPEs in local storage and proceeds with execution after all worker SPEs have signaled completion of the loop.

\subsubsection{SPE-SPE communication}

The SPE threads participating in loop work-sharing constructs are created once upon function off-loading. Communication among SPEs participating in work-sharing constructs is implemented using DMA transfers and the communication structure Pass, depicted in Figure 5.

The Pass structure is private to each thread. The master SPE thread allocates an array of Pass structures. Each member of this array is used for communication with an SPE worker thread. Once the SPE threads are created, they exchange the local addresses of their Pass structures. This address exchange is performed through 
the PPE. Whenever one thread needs to send a signal to a thread on another SPE, it issues an $\mathrm{mfC}$ _put () request and sets the destination address to be the address of the Pass structure of the recipient.

In Figure 6, we illustrate the loop from Figure 3, parallelized with work-sharing among SPE threads. Before executing the loop, the master thread sets the parameters of the Pass structure for each worker SPE and issues one $\mathrm{mfc}$ _put () request per worker. This is done in send_to_spe(). Worker $i$ uses the parameters of the received Pass structure and fetches the data needed for the loop execution to its local storage (function fetch_data()). After finishing the execution of its portion of the loop, a worker sets the res parameter in the local copy of the structure Pass and sends it to the master, using send_to_master(). The master accumulates the results from all workers and commits the sum to main memory.

Immediately after calling send_to_spe(), the master participates in the execution of the loop. The master tends to have a slight head start over the workers. The workers need to complete several DMA requests before they can start executing the loop, in order to fetch the required data from the master's local storage or shared memory. In fine-grained off-loaded functions such as those encountered in RAxML, load imbalance between the master and the workers is noticeable. To achieve better load balancing, we set the master to execute a slightly larger portion of the loop. A fully automated and adaptive implementation of this purposeful load unbalancing is obtained by timing idle periods in the SPEs across multiple invocations of the same loop. The collected times are used for tuning iteration distribution in each invocation, in order to reduce idle time on SPEs.

Table 2 shows the execution times of RAxML with one layer of loop-level parallelism exploited in the off-loaded functions. We execute one bootstrap of RAxML, to isolate the impact of looplevel parallelism. The number of iterations in each parallelized loop depends on the alignment length. For the 42_SC input file, the number of iterations in each parallelized loop is 228 .

\begin{tabular}{|l|l|}
\hline 1 worker, 1 bootstrap, no LLP & $28.71 \mathrm{~s}$ \\
\hline 1 worker, 1 bootstrap, 2 SPEs used for LLP & $20.83 \mathrm{~s}$ \\
\hline 1 worker, 1 bootstrap, 3 SPEs used for LLP & $19.37 \mathrm{~s}$ \\
\hline 1 worker, 1 bootstrap, 4 SPEs used for LLP & $18.28 \mathrm{~s}$ \\
\hline 1 worker, 1 bootstrap, 5 SPEs used for LLP & $18.10 \mathrm{~s}$ \\
\hline 1 worker, 1 bootstrap, 6 SPEs used for LLP & $20.52 \mathrm{~s}$ \\
\hline 1 worker, 1 bootstrap, 7 SPEs used for LLP & $18.27 \mathrm{~s}$ \\
\hline 1 worker, 1 bootstrap, 8 SPEs used for LLP & $24.4 \mathrm{~s}$ \\
\hline
\end{tabular}

Table 2. Execution time of RAxML when loop-level parallelism (LLP) is used in one bootstrap, across SPEs. The input file is 42_SC.

The results in Table 2 suggest that using up to five SPEs for loop parallelization achieves speedup over loop execution using one SPE. The maximum speedup is 1.58. Using five or more SPE threads for loop parallelization decreases efficiency. The reasons for the seemingly low speedup are the non-optimal coverage of loop-level parallelism (less than $90 \%$ of the original sequential code), the fine granularity of the loop for the specific problem size, and the fact that the parallel execution of the loops introduces global reductions, which constitute a synchronization bottleneck. Higher speedup from LLP in a single bootstrap can be obtained with larger input data sets. Alignments that have a larger number of nucleotides per organism have more loop iterations to distribute across SPEs [25].

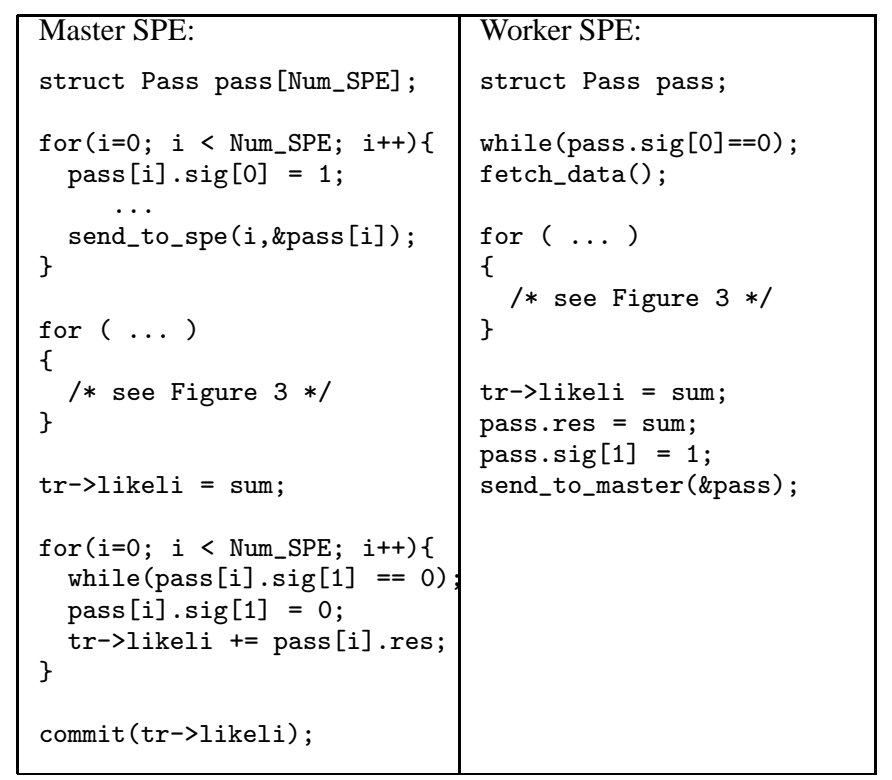

Figure 6. Parallelization of the loop from function evaluate () of RAxML, shown in Figure 3. The left side depitcs the code executed by the master SPE, while the right side depitcs the code executed by a worker SPE. Num_SPE represents the number of SPE worker threads.

\subsection{Adaptive Scheduling of Task-Level and Loop-Level Parallelism}

No single parallelization technique provides the best performance in all possible situations on Cell, a result which is expected, given the variable degree of parallelism available in different components of parallel workloads and the heterogeneity of the Cell architecture.

We implemented a unified dynamic parallelization strategy, which exploits multiple layers of parallelism, by mixing and matching EDTLP with loop-level parallelization, under the control of the run-time system. We name this scheduling strategy multigrain parallelism scheduling (MGPS). The goal of MGPS is to exploit the best of two worlds (TLP and LLP), in response to workload characteristics. MGPS changes parallelization strategies and execution policies on the fly, while the program executes.

To illustrate the need for selectively combining TLP and LLP, we conduct a set of experiments, in which we generate a varying number of bootstraps in RAxML, ranging from 1 to 128, and apply static EDTLP and hybrid EDTLP-LLP parallelization schemes. When LLP is used, each loop uses two or four SPEs, and the PPEs can execute four or two concurrent bootstraps respectively, using EDTLP. This leads to a static multigrain scheme (EDTLPLLP), where LLP is activated when four or less MPI processes are active on the PPE. When LLP is deactivated, we use EDTLP to off-load to all 8 SPEs. The combination of LLP and EDTLP in the static multigrain model is not our final MGPS scheme, since it lacks dynamicity and assumes prior knowledge of runtime program properties. We are using it solely for illustrative purposes.

Figure 7 summarizes the results with a varying number bootstraps. The $x$-axis corresponds to the number of performed bootstraps, and the $y$-axis to the execution time in seconds. We compare the EDTLP-LLP and EDTLP schemes.

As expected, the hybrid model outperforms EDTLP when up to 4 bootstraps are executed, since only a combination of EDTLP and LLP can use more than 4 SPEs simultaneously (see Section 5.2). With 5 to 8 bootstraps, EDTLP activates 5 to 8 SPEs solely for 
(a)

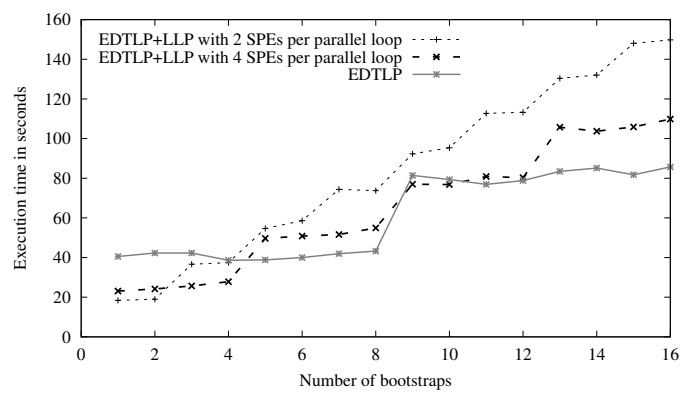

(b)

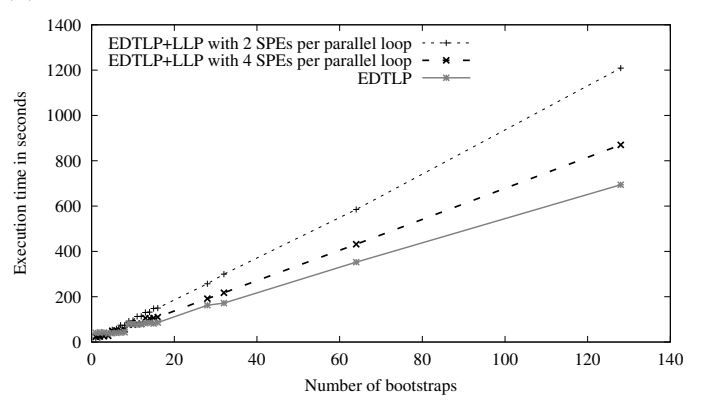

Figure 7. Comparison between the static EDTLP-LLP and EDTLP scheduling schemes. The input file is 42_SC. The number of ML trees created is (a) 1-16, (b) 1-128.

task-level parallelism, leaving room for loop-level parallelism on at most 3 SPEs. This proves to be unnecessary, since the parallel execution time is dominated by the length of the non-parallelized off-loaded tasks that remain on at least one SPE. The hybrid model can simultaneously perform only 2 or 4 bootstraps. Therefore, EDTLP outperforms the hybrid model for 5 to 8 bootstraps. In the range between 9 and 12 bootstraps, combining EDTLP and LLP selectively, so that the first 8 bootstraps execute with EDTLP and the last 4 bootstraps execute with the hybrid scheme is the best option. Note that this scheme is application-specific and requires an oracle to dictate the runtime system when to use EDTLP or EDTLP combined with LLP. Note also that the difference between EDTLP and the hybrid scheme (EDTLP for less than 8 bootstraps, EDTLP-LLP for 9 to 12 bootstraps) will be smaller with 9 to 12 total bootstraps, than with 1 to 4 total bootstraps. In the former case LLP covers up to $11 \%$ (for 9 bootstraps), to $33 \%$ (for 12 bootstraps) of the parallel computation, whereas in the latter case there are always enough SPEs so that the entire parallel computation benefits from LLP.

EDTLP becomes again the best choice with 13 to 16 bootstraps, by the same argument that justifies its superior performance with 5 to 8 bootstraps. As the number of bootstraps increases, the occasional benefit from LLP diminishes, since execution time is dominated by task-level parallelism.

Our experimental observations point to the direction of a dynamic and adaptive user-level scheduler to benefit from multigrain parallelism on Cell. We implemented MGPS, a dynamic scheduler, and tested its performance with RAxML. MGPS extends the EDTLP scheduler with an adaptive processor-saving policy. The scheduler is distributed, and it is executed by every MPI process in the application. MGPS is invoked upon requests for task offloading (arrivals) and upon completion of off-loaded tasks (departures). Initially, upon arrivals, the scheduler conservatively assigns one SPE to each off-loaded task, anticipating that the degree of TLP is sufficient to use all SPEs. Upon a departure, the scheduler checks the degree of task-level parallelism exposed by each MPI process (we will call it $U$ ), i.e. how many discrete tasks were off-loaded to SPEs while the departing task was executing. This number reflects the history of SPE utilization from TLP and is used to switch between the EDTLP policy and the EDTLP-LLP policy. If $U \leq 4$, and $T$ is the number of tasks waiting for off-loading, the scheduler activates LLP with $\left\lfloor\frac{8}{T}\right\rfloor$ SPEs assigned to the parallel loops of each task, if any. If $U>4$, the scheduler retains the EDTLP policy, or deactivates LLP, if LLP was previously activated.

The scheduler is sensitive to the length of the history of TLP, maintained to calculate $U$. As a heuristic, we maintain a history of 800 scheduler invocations. This gives the scheduler the opportunity of a hysteresis of up to 800 off-loaded tasks, before deciding whether to activate LLP. The MPI process that completes the 800th, 1600th, ..., task, evaluates $U$ and signals all other processes to release the idle SPEs, i.e. all SPEs that were not used during the last window of 800 off-loads. Depending on the value of $U$, the scheduler triggers or deactivates LLP. The implementation of the scheduler is facilitated with a shared arena established between MPI processes, to exchange signals and keep track of busy and idle SPEs at any scheduling point (arrivals and departures).

Switching between EDTLP and LLP is enabled by maintaining dual copies of each off-loaded function which includes at least one parallel loop. In the complete adaptive scheduling scheme, each off-loaded function has two or three copies, one PPE copy, one non parallelized SPE copy, and, if the function encapsulates parallel loops, a parallelized SPE copy. Having multiple executable copies of functions increases the total size of the PPE and the SPE code. However, the use of multiple copies reduces the use of conditionals, which are very expensive on the SPEs.

A drawback of the MGPS scheduler is that it initially needs to monitor several off-loading requests from MPI processes, before making a decision for increasing or throttling LLP. If the offloading requests from different processes are spaced apart, there may be extended idle periods on SPEs, before adaptation takes place. In practice, this problem appears rarely, first because applications spawn parallelism early in the code and this parallelism can be directly off-loaded to SPEs, and second because parallelism is typically spawned in bursts from all MPI processes. MGPS handles well applications with static loop-level parallelism as well as applications with static hybrid parallelism, such as MPI/OpenMP applications. To schedule applications that do not off-load enough tasks to trigger adaptation, the scheduler uses timer interrupts.

We compare MGPS against the EDTLP scheduler and the static hybrid (EDTLP-LLP) scheduler, which uses an oracle for the future to guide decisions between EDTLP and EDTLP-LLP. Figure 8 shows the execution times of the MGPS, EDTLP-LLP and EDTLP schedulers with various RAxML workloads. The $x$-axis shows the number of bootstraps, while the $y$-axis shows execution time.

We observe benefits from using MGPS for up to 28 bootstraps, where LLP can be exploited by the scheduler in up to 4 concurrent bootstraps. Beyond 28 bootstraps, MGPS converges to EDTLP, and both are increasingly faster than the static multigrain EDTLPLLP scheme, as the number of bootstraps increases. The loop-level parallel code in MGPS incurs additional overhead for loading the parallel loop code on idle SPEs, potentially replacing the image of an earlier off-loaded function on the SPEs and scheduling the loop. Code replacements occur whenever the runtime system needs to switch between a version with parallelized loops and a version of the same function without parallelized loops, or vice versa. This overhead is not noticeable in overall execution time. Somewhat to our surprise, the overhead is lower than the overhead of using conditionals to select between versions of each function loaded 
(a)

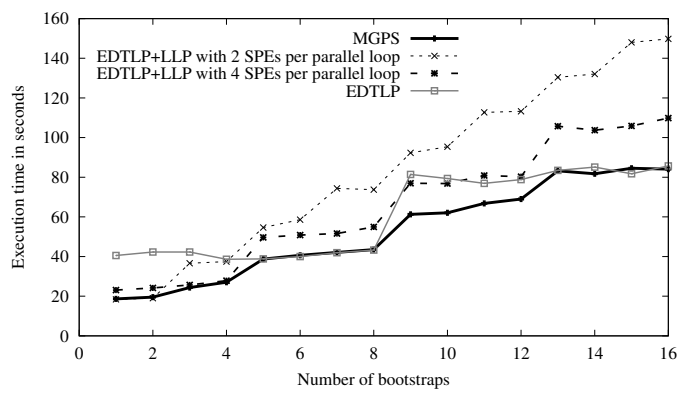

(b)

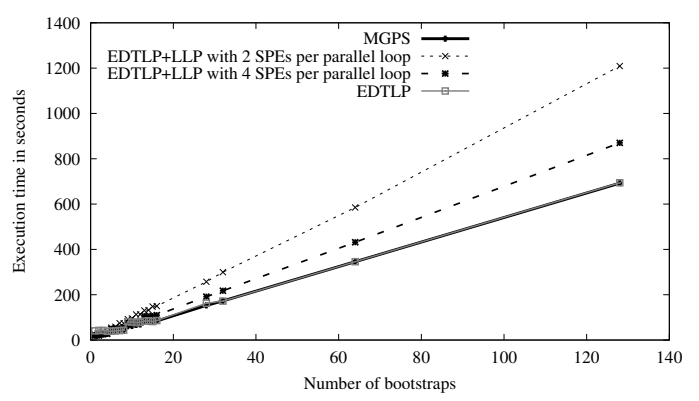

Figure 8. Comparison between the MGPS, EDTLP and static EDTLP-LLP schedulers. The input file is 42_SC. The number of ML trees created is (a) 1-16, (b)1-128. The curves of MGPS and EDTLP overlap completely in (b).

in the same SPE code image. This is an after-effect of the slow handling of branches on the SPEs.

\subsection{Parallelizing Across Multiple Cells}

Figure 9 shows the performance of the MGPS, EDTLP-LLP and the EDTLP schedulers with RAxML on two Cell processors that reside on a single blade. We use the same input file (42_SC) as in the single-processor experiments. The results are qualitatively identical to the results obtained with one Cell processor. The EDTLPLLP model performs better with up to 8 bootstraps, since 8 additional SPEs are available across the two Cells for LLP. Beyond 8 bootstraps, task-level parallelism dominates and EDTLP performs better. MGPS outperforms both EDTLP-LLP and EDTLP.

The reader may point out that since RAxML needs 100 to 1,000 bootstraps for real-world biological analysis, multigrain parallelization is obsolete. Our evaluation indicates that with more than 100 bootstraps, EDTLP is clearly the best option. The results of parallelization across two Cell processors provide a counterargument. For a fixed number of bootstraps, two Cells deliver almost twice the performance of one Cell. As the application is scaled to multiple Cell Processors in the same blade or across blades, running fewer bootstraps per Cell is better than clustering bootstraps in as few Cells as possible. With 100 bootstraps, MGPS with multigrain (EDTLP-LLP) parallelism will outperform plain EDTLP if the bootstraps are distributed between four or more dualCell blades. Taking into account future system scaling, the MGPS scheme is justified in the range of interesting problem sizes for RAxML, even at modest system scales.

\subsection{Comparison of Cell with Other Processors}

As a last point in our evaluation, we compare the performance of the Cell implementation of RAxML and the MPI implementation (a)

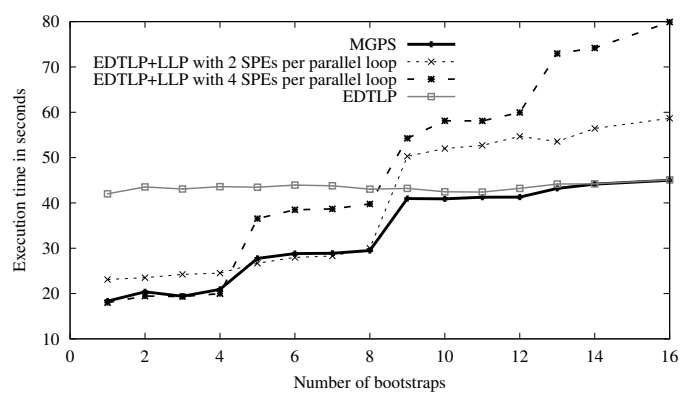

(b)

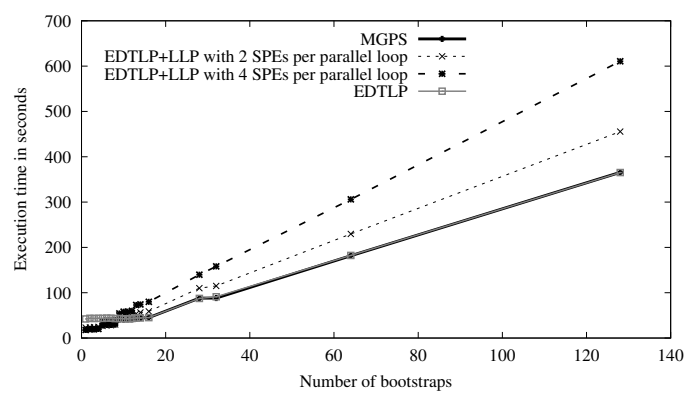

Figure 9. Comparison between MGPS, EDTLP and EDTLP-LLP on two Cell processors. The input file is 42_SC. The number of ML trees created is (a) 1-16, (b) 1-128. The curves of MGPS and EDTLP overlap completely in (b).

of RAxML on systems with commercial multicore and SMT microprocessors. More specifically, we ran RAxML on two platforms:

- A system with two Intel Xeon processors with Hyper-threading technology (2-way SMT), running at $2 \mathrm{GHz}$, with $8 \mathrm{~KB}$ L1-D cache, 512KB L2 cache, and 1MB L3 cache.

- A system with one 64-bit Power5 processor. The Power5 is a quad-thread, dual-core processor with dual SMT cores running at $1.65 \mathrm{GHz}, 32 \mathrm{~KB}$ of L1-D and L1-I cache, $1.92 \mathrm{MB}$ of L2 cache, and $36 \mathrm{MB}$ of $\mathrm{L} 3$ cache.

For all experiments, we use 42_SC as an input file. Figure 10 illustrates execution time versus the number of bootstraps. While conducting the experiments on IBM Power5, we use both cores, and on each core we use both SMT execution contexts, i.e. a total of four MPI processes runs on the Power5 processor. Since one Intel Xeon processor has only two execution contexts, we use two Intel Xeon processors (lying on a 4-way SMP Dell PowerEdge 6650 server), and on each processor we activate both execution contexts. This modification stirs the comparison in favor of the Xeon.

One Cell processor clearly outperforms the Intel Xeon by a large margin, even if two Xeons are used to run RAxML with the same problem size. Cell performs slightly $(5-10 \%)$ better than the IBM Power5, once the problem size is scaled to 8 or more bootstraps. Although the margin of difference between Cell and Power5 is narrow, Cell has an edge over a general-purpose high-end processor such as Power5, since it also achieves better cost-performance and power-performance ratios.

\section{Conclusions}

We investigated policies and mechanisms pertaining to scheduling multigrain parallelism on the Cell Broadband Engine. We proposed an event-driven task scheduler, striving for higher utilization 
(a)

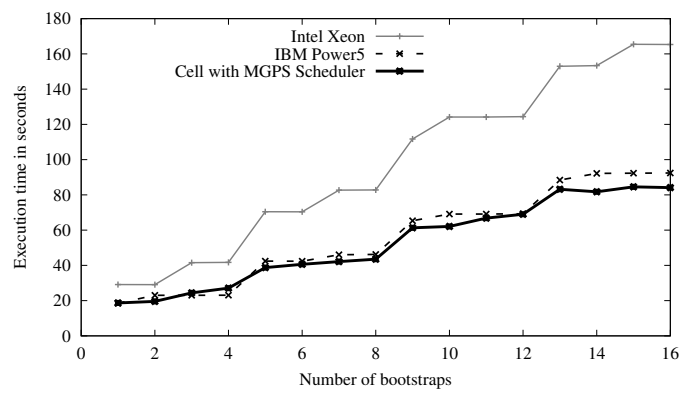

(b)

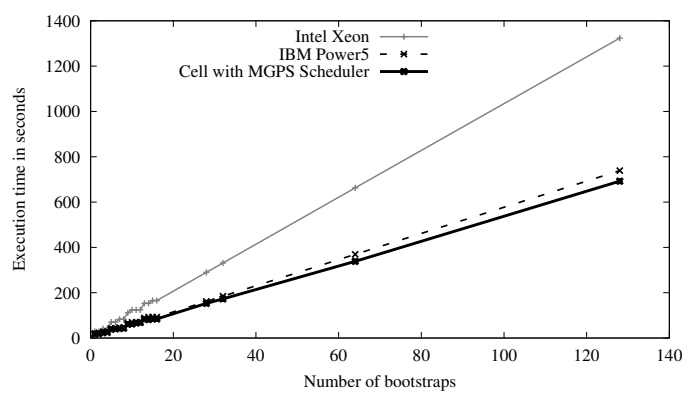

Figure 10. RAxML performance on systems with different multithreaded and multicore microprocessors: A dual-processor system with Intel Xeon HT processors, a single-processor system with an IBM Power5 processors, and a single-processor system with a Cell processor. The number of ML trees created is (a) $1-16$, (b) $1-128$.

of SPEs via oversubscribing the PPE. We have explored the conditions under which loop-level parallelism within off-loaded code can be used. We have also proposed a comprehensive scheduling policy for combining task-level and loop-level parallelism autonomically within MPI code, in response to workload fluctuation. Using a bio-informatics code with inherent multigrain parallelism as a case study, we have shown that our user-level scheduling policies outperform the native OS scheduler by a factor of 2.6.

Our MGPS scheduler proves to be responsive to small and large degrees of task-level and data-level parallelism, at both fine and coarse levels of granularity. This kind of parallelism is commonly found in optimization problems where many workers are spawned to search a very large space of solutions, using a heuristic. RAxML is representative of these applications. MGPS is also appropriate for adaptive and irregular applications such as adaptive mesh refinement, where the application has task-level parallelism with variable granularity (because of load imbalance incurred while meshing subdomains with different structural properties) and, in some implementations, a statically unpredictable degree of task-level parallelism (because of non-deterministic dynamic load balancing which may be employed to improve execution time). N-body simulations and ray-tracing are applications that exhibit similar properties and can also benefit from our scheduler. As a final note, we observe that MGPS reverts to the best static scheduling scheme for regular codes with a fixed degree of task-level parallelism, such as blocked linear algebra kernels.

To simply port RAxML to Cell, we added 1000 lines of code to the application. To optimize the off-loaded code and to implement the SPE-SPE communication code used for loop-level parallelism, we needed an additional 900 lines of code. To implement the MGPS scheduler we added 280 lines of code. The scheduler is portable and we have already deployed it in PBPI, another computational phylogeny package, based on Bayesian inference.

In future work, we intend to incorporate memory-related criteria into our SPE scheduling policies. RAxML simplified the memory management problem, since the major off-loaded functions have small memory footprints and leave enough space for data processing on the SPEs. At the same time, RAxML exhibits a very simple data access pattern, with little sharing of data between tasks loaded on SPEs. We intend to eliminate the assumption of fixed-size SPE code footprints during exploration of scheduling policies in the future. We also plan to stress test our runtime system more as application codes become available on Cell.

\section{Acknowledgments}

This research is supported by the National Science Foundation (Grant CCR-0346867), the U.S. Department of Energy (Grant DE-FG02-06ER25751), the Swiss Confederation Funding, the Barcelona Supercomputing Center, which granted us access to their Cell blades, and equipment funds from the College of Engineering at Virginia Tech.

\section{References}

[1] PowerPC Microprocessor Family: Vector/SIMD Multimedia Extension Technology Programming Environments Manual. http:// www-306.ibm.com / chips / techlib.

[2] D.A. Bader, B.M.E. Moret, and L. Vawter. Industrial Applications of High-Performance Computing for Phylogeny Reconstruction. In Proc. of SPIE ITCom, volume 4528, pages 159-168, 2001.

[3] D.A. Bader, V. Agarwal, and K. Madduri. On the Design and Analysis of Irregular Algorithms on the Cell Processor: a Case Study on List Ranking. In Proc. of the 21st IEEE/ACM International Parallel and Distributed Processing Symposium, March 2007.

[4] C. Benthin, I. Wald, M. Scherbaum, and H. Friedrich. Ray Tracing on the CELL Processor. Technical Report, inTrace Realtime Ray Tracing GmbH, No inTrace-2006-001 (submitted for publication), 2006.

[5] F. Blagojevic, D. S. Nikolopoulos, A. Stamatakis, and C. D. Antonopoulos. RAxML-Cell: Parallel Phylogenetic Tree Inference on the Cell Broadband Engine. In Proceedings of the 21st IEEE/ACM International Parallel and Distributed Processing Symposium, March 2007.

[6] D. A. Brokenshire. Maximizing the Power of the Cell Broadband Engine Processor: 25 Tips to Optimal Application Performance. IBM developerWorks, jun 2006.

[7] M. Charalambous, P. Trancoso, and A. Stamatakis. Initial Experiences Porting a Bioinformatics Application to a Graphics Processor. In In Proceedings of the 10th Panhellenic Conference on Informatics (PCI 2005), pages 415-425, 2005.

[8] T. Chen, R. Raghavan, J. Dale, and E. Iwata. Cell Broadband Engine Architecture and its First Implementation. IBM developerWorks, Nov 2005.

[9] B. Chor and T. Tuller. Maximum Likelihood of Evolutionary Trees: Hardness and Approximation. Bioinformatics, 21(1):97-106, 2005.

[10] T. Z. DeSantis, P. Hugenholtz, N. Larsen, M. Rojas, E. L. Brodie, K. Keller, T. Huber, D. Dalevi, P. Hu, and G. L. Andersen. Greengenes, a Chimera-Checked 16S rRNA Gene Database and Workbench Compatible with ARB. Appl. Environ. Microbiol., 72(7):5069-5072, 2006.

[11] A. E. Eichenberger et al. Optimizing Compiler for a Cell processor. Parallel Architectures and Compilation Techniques, September 2005.

[12] B. Flachs et al. The Microarchitecture of the Streaming Processor for a CELL Processor. Proceedings of the IEEE International Solid-State Circuits Symposium, pages 184-185, February 2005. 
[13] J. Felsenstein. Evolutionary Trees From DNA Sequences: A Maximum Likelihood Approach. Journal of Molecular Evolution, 17:368-376, 1981.

[14] G. W. Grimm, S. S. Renner, A. Stamatakis, and V. Hemleben. A Nuclear Ribosomal DNA Phylogeny of Acer Inferred With Maximum Likelihood, Splits Graphs, and Motif Analyses of 606 Sequences. Evolutionary Bioinformatics Online, 2006. to be published.

[15] S. Guindon and O. Gascuel. A Simple, Fast, and Accurate Algorithm to Estimate Large Phylogenies by Maximum Likelihood. Syst. Biol., 52(5):696-704, 2003.

[16] N. Hjelte. Smoothed Particle Hydrodynamics on the Cell Broadband Engine. Master's thesis, Umeå University, Department of Computer Science, Jun 2006.

[17] M. Kistler, M. Perrone, and F. Petrini. Cell Multiprocessor Interconnection Network: Built for Speed. IEEE Micro, 26(3), MayJune 2006. Available from http:// hpc.pnl.gov / people / fabrizio / papers / ieeemicro-cell.pdf.

[18] R. E. Ley, J. K. Harris, J. Wilcox, J. R. Spear, S. R. Miller, B. M. Bebout, J. A. Maresca, D. A. Bryant, M. L. Sogin, and N. R. Pace. Unexpected Diversity and Complexity of the Guerrero Negro Hypersaline Microbial Mat. Appl. Envir. Microbiol., 72(5):3685 3695, May 2006.

[19] R.E. Ley, F. Backhed, P. Turnbaugh, C.A. Lozupone, R.D. Knight, and J.I. Gordon. Obesity Alters Gut Microbial Ecology. Proceedings of the National Academy of Sciences of the United States of America, 102(31):11070-11075, 2005.

[20] B. Q. Minh, L. .y Vinh, A. von Haeseler, and H. A. Schmidt. pIQPNNI: Parallel Reconstruction of Large Maximum Likelihood Phylogenies. Bioinformatics, 21(19):3794-3796, 2005.
[21] B. Minor, G. Fossum, and V. To. Terrain Renderin Engine (TRE), http:// www.research.ibm.com / cell / whitepapers / TRE.pdf. May 2005.

[22] F. Petrini, G. Fossum, M. Kistler, and M. Perrone. Multicore Suprises: Lesson Learned from Optimizing Sweep3D on the Cell Broadbend Engine.

[23] C.E. Robertson, J.K. Harris, J.R.Spear, and N.R. Pace. Phylogenetic Diversity and Ecology of Environmental Archaea. Current Opinion in Microbiology, 8:638-642, 2005.

[24] A. Stamatakis, T. Ludwig, and H. Meier. Raxml-iii: A Fast Program for Maximum Likelihood-Based Inference of Large Phylogenetic Trees. Bioinformatics, 21(4):456-463, 2005.

[25] A. Stamatakis, M. Ott, and T. Ludwig. Raxml-omp: An Efficient Program for Phylogenetic Inference on SMPs. In Proc. of PaCT05, pages 288-302, 2005.

[26] A. Stamatakis. RAxML-VI-HPC: Maximum Likelihood-Based Phylogenetic Analyses With Thousands of Taxa and Mixed Models. Bioinformatics, page btl446, 2006.

[27] Alias Systems. Alias Cloth Technology Demonstration for the Cell Processor, http:// www.research.ibm.com / cell / whitepapers / alias_cloth.pdf. 2005.

[28] S. Williams, J. Shalf, L. Oliker, S. Kamil, P. Husbands, and K. Yelick. The Potentinal of the Cell Processor for Scientific Computing. ACM International Conference on Computing Frontiers, May 3-6 2006.

[29] D. Zwickl. Genetic Algorithm Approaches for the Phylogenetic Analysis of Large Biological Sequence Datasets under the Maximum Likelihood Criterion. PhD thesis, University of Texas at Austin, April 2006. 